\title{
Biofuel Cells and Bioelectrochemical Systems
}

\section{Abhijeet P Borole ${ }^{1,2 *}$}

${ }^{1}$ Oak Ridge National Laboratory, Biosciences Division, Oak Ridge, TN 37831, USA

${ }^{2}$ University of Tennessee, Department of Chemical and Biomolecular Engineering, Center for Environmental Biotechnology, Knoxville, TN 37996, USA

"The submitted manuscript has been authored by UT-Battelle, LLC, under Contract No. DE-AC05-00OR22725 with the U.S. Department of Energy. The United States Government retains and the publisher, by accepting the article for publication, acknowledges that the United States Government retains a non-exclusive, paid-up, irrevocable, world-wide license to publish or reproduce the published form of this manuscript, or allow others to do so, for United States Government purposes."

This special issue on biofuel cells and bioelectrochemical systems focuses on advances in microbial fuel cells (MFCs) and electrolysis cells, while introducing some new concepts facilitating practical application of these systems. In order to demonstrate feasibility of bioelectrochemical systems, one needs to compare their performance and costs with existing alternatives. The paper by Huggins et al., provides a simple comparison of MFCs vs. aerobic oxidation pits, which are commonly used for treatment of organic wastes at a low cost. The work reports results from a 15L scale unit, which demonstrates the effectiveness of MFCs and identifies an interesting configuration for development of low cost systems with application for treatment of low value streams.

The working principle of a microbial fuel cell inherently requires movement of positive ions from the anodefluid to the cathode due to electrons being consumed at the cathode. A modification of a typical MFC can also allow separation of positive, as well as negative charges to respective electrodes. Gude et al., report the current status of such cells termed as microbial desalination cells and discuss their limitations and potential strategies to overcome them. They also introduce concepts based on photosynthetic MFCs to improve removal of nutrients from wastewater as a means of resource recovery, while also sequestering carbon dioxide.

A paper by Babu et al. focuses on investigations of process parameters on operation of microbial electrolysis cells (MEC) for hydrogen production from synthetic organic acids. The effect of pretreatment of the inoculum on hydrogen production is reported, which is a unique way to minimize methanogenesis in MEC, thereby improving yield of hydrogen.

One of the factors limiting power production in MFCs is the cathode performance. Ichihashi and Hirooka report on studies of MFC cathodes subjected to various post-operation treatments to understand the reasons for deterioration of its performance over time. The restoration of performance via removal of specific water soluble ions is discussed.

Bioelectrochemical losses in biofuel cells and other systems have limited advancement of this technology to the next level. One tool that has shown great utility in understanding the limitations of bioelectrochemical systems is electrochemical impedance spectroscopy (EIS). A review paper by Sekar and Ramasamy outline the current status of our understanding of these systems based on EIS studies. They discuss the fundamental basis of the technique, as well as the specific modes in which this has been applied. EIS has a long history of application in conventional fuel cells and biosensors, many of which have not been applied to bioelectrochemical systems. Sekar and Ramasamy discuss a few advanced modes of application and highlight the scope of these methodologies to develop BES systems further.

The basis of impedance and the equivalence of electrochemical parameters, including electrolyte resistance, double layer capacitance, polarization resistance, diffusion and charge transfer resistance to relevant MFC variables are discussed. The role of mediators vs. direct electron transfer in microbial fuel cells and limitations to bioelectrochemical processes occurring in these systems is outlined. They have compiled the literature results on the effect of various process parameters on microbial fuel cell performance using EIS as a tool and evaluate how equivalent circuit models describe them.

Electrosynthesis using microbes for production of fuels and chemicals has been an active area of research within the field of bioelectrochemical systems. The use of enzymes for electrosynthesis, however, has not been reported extensively. Dominguez-Benetton et al., introduce the concept of enzyme-based electrosynthesis and explore the possibilities using enzyme based pathways for production of bioenergy and bioproducts. They review the recent advances in enzyme fuel cells and pry out the findings from this body of literature applicable to enzymatic electrosynthesis. The discoveries of direct and novel mediated electron transfer in the area of enzyme, as well as microbial fuel cells, may have significant implications to direct synthesis of fuels and chemicals using enzymes. The use of enzyme cascades can enable incorporation of multi-step pathways into microchanneled or compartmentalized micro-reactors. Production of target products can be directed in such reactors by controlling the biocatalyst concentrations based on the respective specific enzyme conversion rates, minimizing accumulation of intermediates and increasing yield of the target products.

*Corresponding author: Dr. Abhijeet P Borole, Oak Ridge National Laboratory,
Biosciences Division, Oak Ridge, TN 37831, USA, Fax: (865) 241 1555; E-mail: borolea@ornl.gov

Received November 13, 2013; Accepted November 13, 2013; Published November 18, 2013

Citation: Borole AP (2013) Biofuel Cells and Bioelectrochemical Systems. J Microb Biochem Technol S6: e001. doi:10.4172/1948-5948.S6-e001

Copyright: ( 2013 Borole AP. This is an open-access article distributed under the terms of the Creative Commons Attribution License, which permits unrestricted use, distribution, and reproduction in any medium, provided the original author and source are credited 